\title{
On Two Cryogenic Systems of High Purity Germanium Detector
}

\author{
Jianyong Zhang, Xiao Cai, Xiaohu Mo \\ Institute of High Energy Physics, Chinese Academy of Science, Beijing, China. \\ Email: jyzhang@ihep.ac.cn, caixiao@ihep.ac.cn,moxh@ihep.ac.cn \\ Received August $20^{\text {th }}, 2013$; revised September 21 $1^{\text {st }}, 2013$; accepted September $28^{\text {th }}, 2013$ \\ Copyright (C) 2013 JianYong Zhang et al. This is an open access article distributed under the Creative Commons Attribution License, \\ which permits unrestricted use, distribution, and reproduction in any medium, provided the original work is properly cited.
}

\begin{abstract}
Two cryogenic systems of high purity germanium detector, liquid nitrogen and mechanical cooler, are expounded, together with explanations of merits and demerits for each kind of cooling methods. The resolutions of detector to the characteristic lines of ${ }^{152} \mathrm{Eu}$ under different cooling conditions are studied. The laboratory results indicate that the mechanical cooler (X-Cooler II) is an ideal replacement candidate for the liquid nitrogen cooling system that is being utilized by BEMS at BEPC-II.
\end{abstract}

Keywords: HPGe Detector; Liquid Nitrogen; X-Cooler II

\section{Introduction}

The upgraded Beijing electron-positron collider (BEPCII) is a $\tau$-charm factory with a center mass of energy ranging from 2.0 to $4.6 \mathrm{GeV}$ and a design peak luminosity of $10^{33} \mathrm{~cm}^{-2} \cdot \mathrm{s}^{-1}[1,2]$. The upgraded Beijing spectrometer detector (BES-III) with high efficiency and good resolution both for charged and neutral particles was constructed and started data taking in 2008 [3]. The BES-III research region covers charm physics, charmonium physics, spectroscopy of light hadrons and $\tau$-lepton physics [4].

After vast amounts of data are acquired and analyzed, in physics analyses, the statistical uncertainty becomes smaller and smaller, the systematic uncertainty plays more and more prominent roles [5-7], one of which is the uncertainty due to beam energy. To decrease the uncertainty of beam energy, a high accuracy beam energy measurement system (BEMS) [8-11] has been constructed at the north crossing point of the BEPC-II, which is of great importance for many physics analyses at BES-III, such as $\tau$ mass measurement, charmonium resonance scans, and determination of the branching ratio with the uncertainty at the level of $1 \%-2 \%$. The working principle of BEMS can be recapitulated as follows [12]: firstly, the laser source provides the laser beam and the optics system focuses the laser beam and guides it to make head-on collisions with the electron (or positron) beam in the vacuum pipe, after that, the backscattering high energy photon will be detected by the High Purity Germanium (HPGe) detector, which is the key instrument of BEMS. The accuracy of beam energy depends solely on the detection results of HPGe detector.

The crucial condition for HPGe detector functioning properly is the low temperature [13-15] that allows the single crystalline germanium structure of the detector to operate as a diode, which produces current proportional to the energy deposited by a gamma ray interacting with that structure. Two approaches are usually employed to get the temperature below 100 kelvins (K): liquid nitrogen $\left(\mathrm{LN}_{2}\right)$ and mechanical cooler. The former is adopted for cooling the HPGe detector at BEMS. However, one conspicuous drawback to the use of $\mathrm{LN}_{2}$ owes to the time required to fill the dewar. The experience at BEPC-II is a half-hour filling once a week for HPGe detector of BEMS. During the perfect data taking process of BES-III, the precious time has to be consumed for $\mathrm{LN}_{2}$ filling.

Mechanical cooler is an ideal replacement for $\mathrm{LN}_{2}$ because it can provide continuous cooling as long as electricity is available. Only concern here is the resolution of HPGe detector with mechanical cooler, since the range of cooling temperature for mechanical cooler is from 85 to $105 \mathrm{~K}$, which is a little bit higher than that for $\mathrm{LN}_{2}$ (the boiling temperature for $\mathrm{LN}_{2}$ is $77 \mathrm{~K}$ at standard temperature and pressure). Therefore, in this monograph besides the detailed description of two cryogenic systems, the resolutions with distinctive cooling methods are meas- 
ured with the radiation source of ${ }^{152} \mathrm{Eu}$. The laboratory results confirm the replacement advantage of the mechanical cooler over $\mathrm{LN}_{2}$ cooling, which is being used at BEPC-II.

\section{Two Cryogenic Systems}

\subsection{HPGe Detector}

Germanium detectors are semiconductor diodes having a P-I-N structure in which the intrinsic region is sensitive to ionizing radiation, particularly X-rays and $\gamma$-rays. There are three types of reaction which happen in germanium semiconductor, that is, photoelectric effect, Compton scattering and pair production $[16,17]$. The latter two processes dominate when the energy of injection photon is greater than $1 \mathrm{MeV}$. The germanium has a net impurity level of around $10^{10}$ atoms $/ \mathrm{cm}^{3}$, so that with moderate reverse bias voltage, the intrinsic region that is the entire volume between the electrodes is depleted, and an electric field extends across this active region. When photons interact with the material within the depleted volume of a detector, charge carriers (holes and electrons) are produced and are swept by the electric field to the $\mathrm{P}$ and $\mathrm{N}$ electrodes. This charge, which is in proportion to the energy deposited in the detector by the incoming photon, is converted into a voltage pulse by an integral charge sensitive preamplifier. Subsequent amplification and pulse height analysis add the pulse to accumulated histogram which eventually becomes the characteristic spectrum of the source.

There are two kinds of HPGe detectors, n-type and p-type. Since the latter is utilized by BEMS at BEPCII, the similar coaxial p-type HPGe detector [18] manufactured by ORTEC is adopted, whose model is GEM20180-P. It has diameter of $54.0 \mathrm{~mm}$ and length of 50.2 $\mathrm{mm}$, the relative efficiency is $23 \%$. The energy resolution for the $1.33 \mathrm{MeV}$ line of ${ }^{60} \mathrm{Co}$ is $1.67 \mathrm{keV}$.

There are two types of capsule for the HPGe detector [19]: the streamline and the PopTop structure. In the streamline system, the germanium crystal, pre-amplifier and high voltage filter are packaged in the capsule, the capsule and the cryostat share the same vacuum, the detector capsule is not demountable. For the PopTop system, the capsule has its own vacuum; it can be mounted on any of the available cryostats. It is convenient for the PopTop structure of HPGe detector to change the cooling method. The cryostat/dewar combinations can be cooled by $\mathrm{LN}_{2}$, or the detector can be cooled using mechanical cooler II, by cold head of cooler coupling with PopTop capsule of detector. For the experiment that follows, the PopTop detector will be used.

\subsection{Liquid Nitrogen Cooling}

The germanium crystal is cooled down to the working temperature by connecting with the thermal transfer device, the cryostat and the extension rod, the latter is dipped into the $\mathrm{LN}_{2}$-full dewar, and the cold is conducted from the dewar to the germanium crystal. To make the heat exchange much easier, the thermal transfer device is usually made of copper. Before insert the cryostat detector into a dewar, a white silicone rubber collar should be placed onto the dewar, so that a gas-tight seal is formed. The silicone rubber contains two stainless steel tubes, which are used for $\mathrm{LN}_{2}$ filling and gas exhaust. During filling, liquid nitrogen enters through either tube, the other tube will exhaust the excess liquid when the dewar is full.

One common filling method uses a standard 30-liter dewar of $\mathrm{LN}_{2}$ as a supply dewar. As shown in Figure 1, a gas-tight fixture, which holds a metal outlet tube and a gas inlet for pressurization, is attached to the supply dewar flange. The metal outlet tube is connected to a short length of plastic tube which serves as the supply hose. This tube carries liquid nitrogen out from dewar bottom. Transfer is effective by pressurizing the dewar at 3 to 5 psi with dry nitrogen gas. The supply dewar or the gas inlet tube must have a pressure relief valve set at 5 psi. One must monitor the $\mathrm{LN}_{2}$ filling continuously, once the dewar is full, the $\mathrm{LN}_{2}$ overflow from the exhausting tube, the transfer process must be terminated immediately by turning off the filling valve. The transfer requires only a few minutes if the gas pressure is not less than 5 psi. During the transfer process, the surface of the supply and exhaust hoses is covered with frost, allow them to thaw completely before removing them from the fill tubes [19].

The $\mathrm{LN}_{2}$ supply system depicted above is called the self-pressurizing system, the pressure produced by the expansion of $\mathrm{LN}_{2}$ to the nitrogen gas due to the evaporation of the $\mathrm{LN}_{2}$. Therefore, about ten hours should be laid aside before the pressure in dewar is high enough to

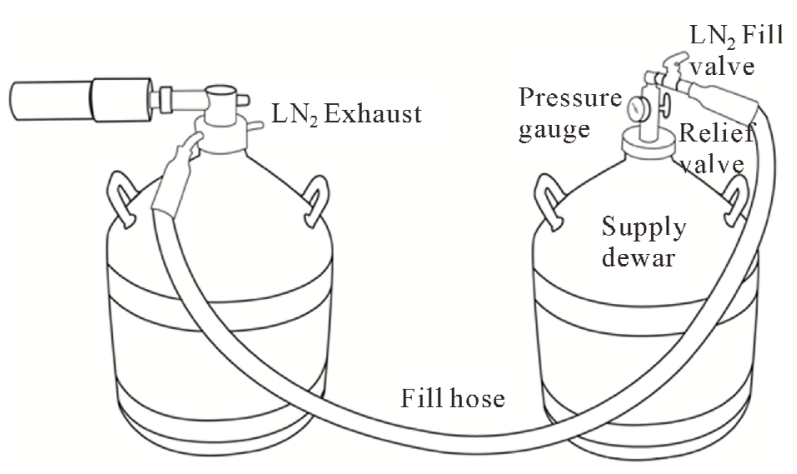

Figure 1. Tubing arrangement for liquid nitrogen transfer. The foam insulated fill hose is used to connect the supply dewar with the working dewar. The pressure gauge displays the pressure of dry nitrogen gas in the supply dewar. The fill valve should be turned off when the transfer process finish, see text for the details. 
press the $\mathrm{LN}_{2}$ into the detector dewar quickly. But if the filling dewar stays without using for a long time, the $\mathrm{LN}_{2}$ will evaporate slowly. Figure 2 shows the dependence of the loss of $\mathrm{LN}_{2}$ on the storage time (the experiment temperature is $26^{\circ} \mathrm{C}$ ). The longer is the storage time, the heavier of $\mathrm{LN}_{2}$ loss. The time-loss dependence is almost linear as shown in Figure 2.

Special attention must be paid for the cooling time, especially when the detector is firstly cooled down. It must be ensure that the boiling off of $\mathrm{LN}_{2}$ will be excessive until the detector element is completely cooled. One can not apply the high voltage to bias the detector before the germanium crystal is cooled for adequate long time. Concretely speaking, six hours is need for the detector used at BEMS. Data taken can be begun till the detector is biased and the voltage is stable. It is worthy of stressing that accidental application of high voltage to a detector which is not fully cold can cause serious damage and even impair the detector thoroughly.

During the data taking period of BES-III, the BEMS is kept running simultaneously. The once-a-week filling schedule is followed to avoid unexpected warm-ups of HPGe detector. This reduces the probability that the temperature of germanium crystal is too high to work. However, such a regular filling schedule is unfavorable by both BES-III detector and BEPC-II accelerator. For the detector, some precious data taking time has to be consumed for refilling $\mathrm{LN}_{2}$; for the accelerator, some time has to be used to recover the preceding good running status. Moreover, when accelerator is under unstable status, accident may be happened and accelerator has to be stopped. This kind of time is an opportunity for $\mathrm{LN}_{2}$ filling. But as we mentioned before, the self-pressurizing technique need additional time for increasing the pressure from the evaporation of the $\mathrm{LN}_{2}$. Therefore, the $\mathrm{LN}_{2}$ has to be filled beforehand. However, the unpredictability of accident usually leaves the full dewar untouched

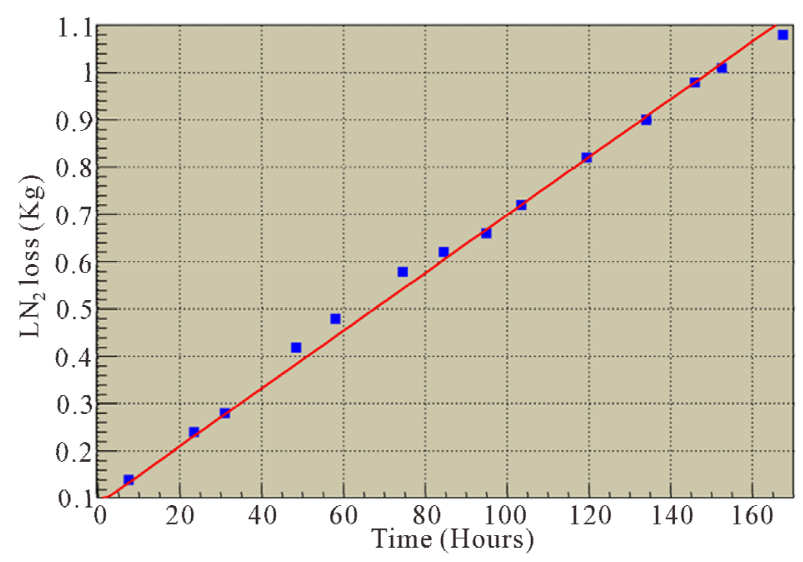

Figure 2. Dependence of the loss of $\mathrm{LN}_{2}$ on the storage time. The points are experiment data while the line is the linear fit result. more than a week, which leads to lots of loss of $\mathrm{LN}_{2}$ as indicated by the time-loss curve in Figure 2.

Besides aforementioned disfavors, users have to face hazards associated with $\mathrm{LN}_{2}$. For persons who transfer, store, and fill $\mathrm{LN}_{2}$, they should avoid skin contact with $\mathrm{LN}_{2}$, which causes frostbite. For the material storing, transfer, and filling $\mathrm{LN}_{2}$, most of which become brittle and fracture when exposed to the $\mathrm{LN}_{2}$, especially the detector cryostat and electronics. In addition, transfer $\mathrm{LN}_{2}$ should be operated in a well ventilated area, although the nitrogen gas is nontoxic; it is possible to cause asphyxiation by displacing air.

\subsection{Mechanical Cooler Cooling}

A cooler is a device used to cool the environment and anything inside it to extremely cold temperatures. Typically used in scientific and engineering applications, it is designed to achieve temperatures well below those reached by standard appliances [20].

To most coolers, gas is typically circulated through a closed cycle to absorb heat from the interior of the device and transfer it to the outside environment. This gas may be hydrogen, helium, or some other gas or mixture of gases. The ability of the device to cool its interior environment depends largely on the thermodynamic properties of the gas circulating through the system. Cooling cycles typically begin with gas being compressed in a compressor. As the compressed gas passes through a heat exchanger, it absorbs heat from the inside of the cooler, thereby cooling anything inside it. When this gas absorbs heat at a constant volume in the heat exchanger, its pressure increases. It expands in volume and its pressure decreases in the next portion of the cycle. Finally, it returns to the compressor, which completes a closed loop through the cycle, and begins to circulate through the cycle again.

Many types of coolers are available with different advantages and features suitable for a wide variety of applications. Common types of coolers include the JouleThomson cooler, the Gifford-McMahon cooler, the Stirling cooler, and so on.

The X-Cooler II is adopted to provide continuous cooling for HPGe detector. It includes a compressor, transfer hose, heat exchanger, and cold head. All parts except for the heat exchanger are visible to user, refer to Figure 3 for each part. The compressor is the box (denoted by the green box in Figure 3) with a standard instrument plug on the rear for connecting AC power. Inside the box are the compressor, a cooling fan, some AC wiring, and the plumbing of the refrigerant system. Attached to the rear panel of the compressor box is the transfer hose, which is made of a stainless steel braid hose, 0.25 inch in diameter, that contains the gas pressure and return lines. Over the stainless steel braid hose is 


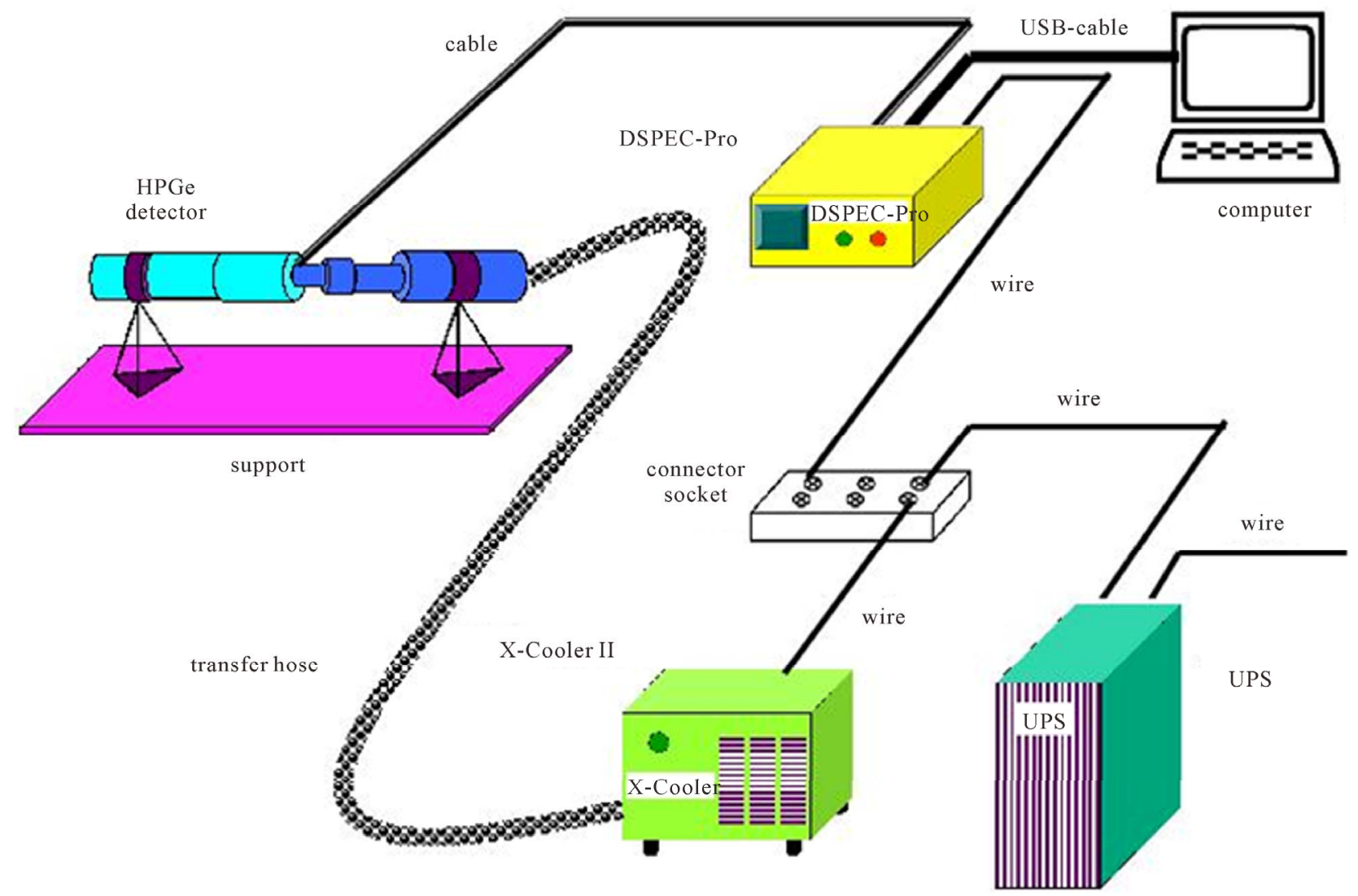

Figure 3. Design cartoon of mechanical cooler system for the HPGe detector that is composed of two parts: data acquisition system and cooling system. The former mainly consists of a DSPEC-pro and a computer. The DSPEC-pro is connected with both the Germanium crystal by detector interface module, and the computer by USB. All data processing is finished by the computer. The cooling system shown here is called X-Cooler II that is composed of a compressor, transfer hose, heat exchanger, and cold head. The heat exchanger is inside the cold head denoted by the dark blue tube, and connected with germanium crystal. The cold head and compressor are connected by the transfer hose. The UPS is added to provide the XCooler II with high quality power supply.

black insulting material and around that is a black plastic protective netting material. The transfer hose is often called "the umbilical cord" and connects the heat exchanger to the compressor box. At the end of transfer hose is the heat exchanger. It is a coiled set of copper and stainless tubing mounted inside the 3-inch diameter stainless tube. It is in this heat exchanger that the gas expansion for necessary cooling takes place.

The heat exchanger is inside the assembly known as the cold head. The cold head (connected with HPGe detector, and denoted by the dark blue tube in Figure 3) also contains the thermal transfer devices and threaded coupling required to attach an ORTEC HPGe detector in a PopTop capsule to the X-Cooler II. Compared with $\mathrm{LN}_{2}$ cooling, $\mathrm{X}$-CoolerII cooling is much simple. Pull out the detector from the dewar and warm the detector to the room temperature, remove the capsule from the cryostat and couple it to the cold head of X-Cooler II. During cooling, the X-Cooler II uses patent technology [21] to remove residual oil and other contaminant, which allows the design to use standard, off-the-shelf compressors.

The length of time it takes the X-Cooler II to cool HPGe detector to an acceptable temperature depends on the size of the detector and the ambient environmental conditions. For the coaxial p-type detector (GEM-20180P) used this experiment, the time between 12 and 14 hours [14] is enough to get to stable operating temperature for data taking.

Comparing with $\mathrm{LN}_{2}$ method, the accommodation of continuous cooling by the X-CoolerII resolves the interrupt annoy for data taking of BES-III, and the safety hazards associated with $\mathrm{LN}_{2}$ are eliminated as well. Nevertheless, there are some hazards for the X-Cooler II to confront with, such as electrical hazard, temperature hazard, and gas hazard, as to which user can find the details of precautions from user's manual [14].

As a matter of fact, an uninterruptible power system (UPS) is usually recommended to be prepared for the electric cooler, which guarantees the power supply. If the $\mathrm{X}$-CoolerII loses power, one can reapply power within 10 
minutes of initial power loss. If the power loss lasts for more than 10 minutes, the system must be warmed to room temperature before re-cooling, and the warm-up time is 24 hours. For the refrigerant is a mixture of gases, it goes through a separation process when the compressor is running. If the compressor is shut off, warm up the cooler to room temperature will allow the refrigerant to equilibrium. If the cooler is started while the refrigerant is still separated, the compressor could be damaged because it is not operating the complete refrigerant mixture.

\section{Resolutions of the Two Cooling Methods}

\subsection{Radiation Source ${ }^{152} \mathrm{Eu}$}

The radiation source adopted is the nuclide ${ }^{152} \mathrm{Eu}$ whose decays is complicated, about $27 \%$ via $\beta$ transitions, followed by photon emission, and $73 \%$ electron capture processes, with subsequent gamma emission. ${ }^{152} \mathrm{Eu}$ is an important gamma-emitter in radionuclide metrology and is used extensively to calibrate gamma-spectrometers for both energy and efficiency. The gamma energy range emitted by ${ }^{152} \mathrm{Eu}$ is wide, the main lines is from hundred $\mathrm{keV}$ to $1.4 \mathrm{MeV}[22,23]$.

\subsection{Setup and Experiment}

The HPGe detector can be classified into two subsystems: cooling system and data acquisition system. The latter (refer to Figure 3) is mainly composed of a DSPEC-pro and a computer. For our experiment, the germanium detector is connected with the ORTEC DSPEC-pro by detector interface module (DIM). The high voltage power supply, amplifier and multi channel analyzer (MCA) are integrated into DSPEC-pro. Then, the DSPEC-pro is plug into a computer by USB. All the control to the detector and parameters set are operated by computer.

A point like ${ }^{152} \mathrm{Eu}$ nuclide is used in this experiment, its characteristic peaks are recorded to monitor the change of HPGe detector resolutions under different cooling conditions. During the experiment, under the two cryogenic systems, the radiation source is placed along the cylindrical center axis of the Ge crystal, and about $1 \mathrm{~cm}$ far away from the top of the germanium detector. A foam plate with $1 \mathrm{~cm}$ thickness is sandwiched between the source and the detector. The whole resolution experiment is divided into two steps and before each step, both ${ }^{137} \mathrm{Cs}$ and ${ }^{60} \mathrm{Co}$ are used for the calibration of the HPGe detector.

The first step of experiment begins with the X-cooler II case, the data of measurement to radiation source are collected from 9:00 Nov. 21 st, to 16:50 Nov. $23 \mathrm{rd}, 2012$. In order to remove the background effect, three days' background data are taken before and after the ${ }^{152} \mathrm{Eu}$ nuclide measurement, respectively.

The second step of experiment turns to $\mathrm{LN}_{2}$ cooling method. The PopTop capsule of the detector is removed from the cold head of the X-cooler II, then connected with the cryostat, and put into a dewar filled with liquid nitrogen. After about six hours cooling, the germanium crystal is cold enough to apply the high voltage to bias the detector. The radiation source experiment is performed from 17:00 Dec. 7 th, to 8:00 Dec. 10 th, 2012.

Also the background data of about two days are taken before and after the measurement of ${ }^{152} \mathrm{Eu}$ under the $\mathrm{LN}_{2}$ cooling, respectively.

\subsection{Results}

The spectra of ${ }^{152} \mathrm{Eu}$ measured by HPGe detector under different cryogenic systems are shown in Figure 4, where removed are the backgrounds that have been normalized using the measurement time. The noise level for both $\mathrm{LN}_{2}$ cooling and $\mathrm{X}$-Cooler II cooling is the same, around $10 \mathrm{keV}$. Lots of characteristic peaks of ${ }^{152} \mathrm{Eu}$ from $122 \mathrm{keV}$ to $1400 \mathrm{keV}$ can be seen clearly, and three typical lines, that are the line 244.7, 688.7, and 1408.0 $\mathrm{keV}$, can be found in Figure 5. Although the shapes of the lines are almost the same, the resolution of the germanium detector using the X-Cooler II is obviously better than that using the $\mathrm{LN}_{2}$ case.

The detailed information of the characteristic peaks is collected in Table 1, where presented are the measured energy of characteristic line, the full width at half maximum (FWHM) and the full width at one-fifth maximum (FW [1/5] M) of each peak, and the ratio of two kinds of resolutions, which is defined as follows:

$$
\mathrm{R}=\frac{\text { resolution of } \mathrm{LN}_{2}}{\text { resolution of } \mathrm{X}-\text { Cooler II }} \text {. }
$$

The maximum relative difference of measured energy of characteristic line for two cryogenic systems is at the level of one per mille, while the resolutions under $\mathrm{X}$ Cooler II cooling is generally $10 \%$ better than those under $\mathrm{LN}_{2}$ cooling (refer to the last two columns of Table 1). These experimental results celebrate the mechanical cooler cooling system as an excellent replacement for liquid nitrogen cooling system.

\section{Conclusions}

The liquid nitrogen cooling system has served BEMS for more than three years and kept the smooth commissioning of HPGe detector under the stable status. The obvious demerit of this cooling system lies in the occupation of some fractions of running time for the regular refilling.

The promising successor of liquid nitrogen cooling is the mechanical cooler, i.e., the X-Cooler II, that can provide the continuous cooling, and the regular interruption of data taking will be exterminated for good. At the same 


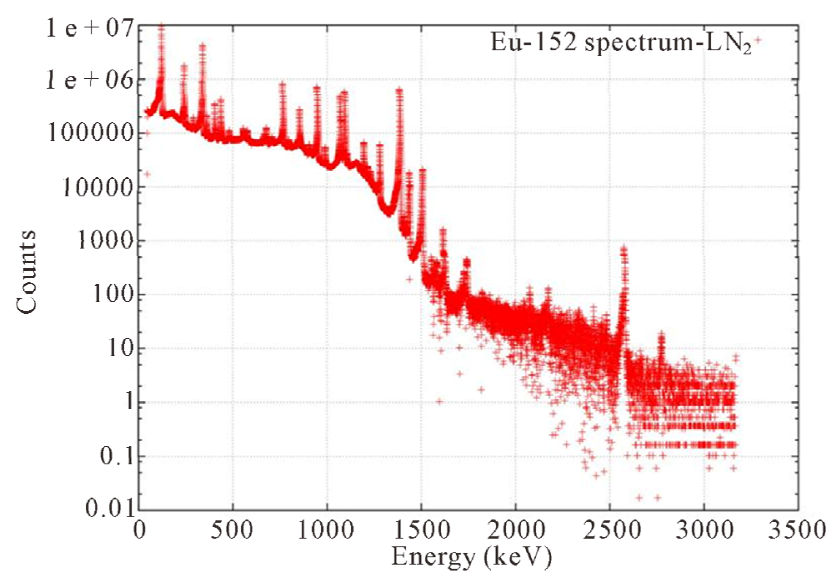

(a) $\mathrm{LN}_{2}$ system

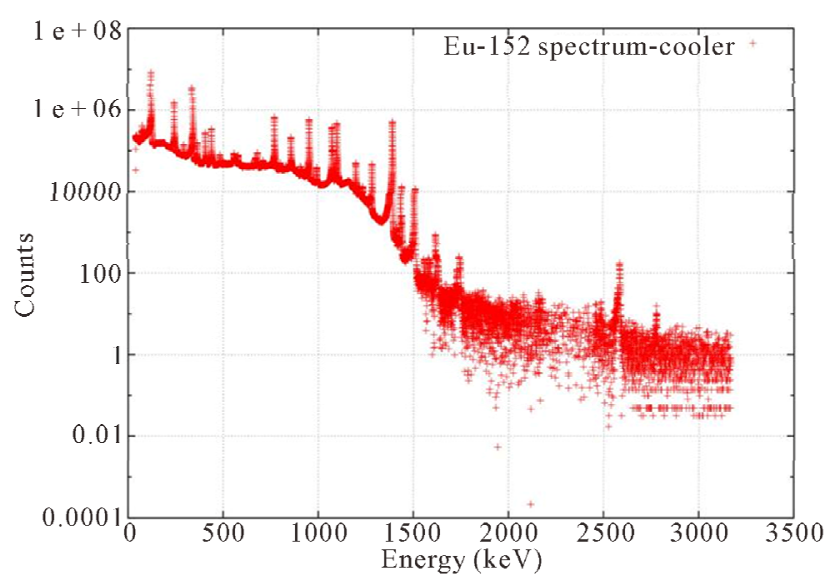

(b) X-Cooler II system

Figure 4. The spectra of ${ }^{152}$ Eu measured by HPGe detector under the cooling of liquid nitrogen (a) and X-Cooler II (b) The normalized background has been subtracted.

Table 1. Detailed information for each line of ${ }^{152}$ Eu nuclide under different cooling condition.

\begin{tabular}{|c|c|c|c|c|c|c|c|c|}
\hline \multirow{2}{*}{$\begin{array}{l}\text { Nomial lines } \\
(\mathrm{keV})[24]\end{array}$} & \multicolumn{3}{|c|}{ Liquid nitrogen (keV) } & \multicolumn{3}{|c|}{ X-Cooler II (keV) } & \multirow{2}{*}{$\begin{array}{c}\mathrm{R} \\
\text { FWHM }\end{array}$} & \multirow{2}{*}{$\begin{array}{c}\mathrm{R} \\
\mathrm{FW}[1 / 5] \mathrm{M}\end{array}$} \\
\hline & Energy & FWHM & $F W[1 / 5] M$ & Energy & FWHM & $F W[1 / 5] M$ & & \\
\hline 121.8 & 121.67 & 1.23 & 2.35 & 121.69 & 1.10 & 1.99 & 1.10 & 1.18 \\
\hline 244.7 & 244.18 & 1.36 & 2.41 & 244.36 & 1.23 & 2.10 & 1.11 & 1.15 \\
\hline 344.3 & 343.63 & 1.47 & 2.57 & 343.92 & 1.32 & 2.26 & 1.11 & 1.14 \\
\hline 411.1 & 410.51 & 1.55 & 2.67 & 410.84 & 1.43 & 2.42 & 1.08 & 1.10 \\
\hline 444.0 & 423.28 & 1.61 & 2.72 & 443.69 & 1.42 & 2.37 & 1.13 & 1.15 \\
\hline 488.7 & 488.01 & 1.60 & 2.70 & 488.45 & 1.46 & 2.47 & 1.10 & 1.09 \\
\hline 586.3 & 585.48 & 1.67 & 2.79 & 586.05 & 1.56 & 2.57 & 1.07 & 1.09 \\
\hline 678.6 & 677.68 & 1.72 & 2.76 & 678.38 & 1.54 & 2.56 & 1.08 & 1.08 \\
\hline 688.7 & 687.71 & 1.75 & 2.90 & 688.45 & 1.63 & 2.61 & 1.07 & 1.11 \\
\hline 778.9 & 777.77 & 1.84 & 2.97 & 778.61 & 1.70 & 2.73 & 1.08 & 1.09 \\
\hline 867.4 & 866.13 & 1.94 & 3.19 & 867.05 & 1.80 & 2.91 & 1.08 & 1.10 \\
\hline 964.0 & 962.72 & 1.99 & 3.20 & 963.78 & 1.87 & 3.01 & 1.06 & 1.06 \\
\hline 1005.1 & 1003.83 & 2.02 & 3.25 & 1004.95 & 1.93 & 3.03 & 1.05 & 1.07 \\
\hline 1085.8 & 1084.40 & 1.99 & 3.14 & 1085.58 & 1.87 & 2.93 & 1.06 & 1.07 \\
\hline 1112.1 & 1110.62 & 2.10 & 3.42 & 1111.85 & 1.97 & 3.16 & 1.07 & 1.08 \\
\hline 1212.9 & 1211.36 & 2.21 & 3.61 & 1212.71 & 2.04 & 3.33 & 1.08 & 1.08 \\
\hline 1299.1 & 1297.41 & 2.27 & 3.67 & 1298.88 & 2.13 & 3.46 & 1.07 & 1.06 \\
\hline 1408.0 & 1406.12 & 2.38 & 3.92 & 1407.71 & 2.22 & 3.61 & 1.07 & 1.09 \\
\hline
\end{tabular}

time, the safety hazards related to liquid nitrogen filling is eliminated as well. Furthermore, the laboratory measurements indicate that the resolution of HPGe detector under the X-Cooler II is even better than that of liquid nitrogen cooling, which is another favorable feature for
BEMS.

The only thing that needs the further confirmation of experiment is the environment effect on the X-Cooler II cooling system, since the conditions under storage ring tunnel is rather different from those of laboratory. More 


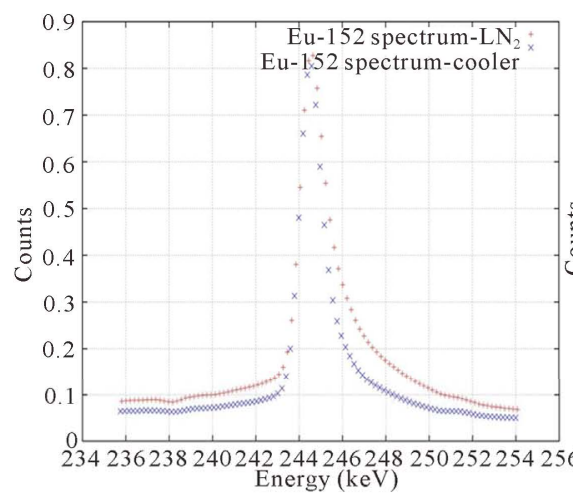

(a) Line $244.7 \mathrm{keV}$

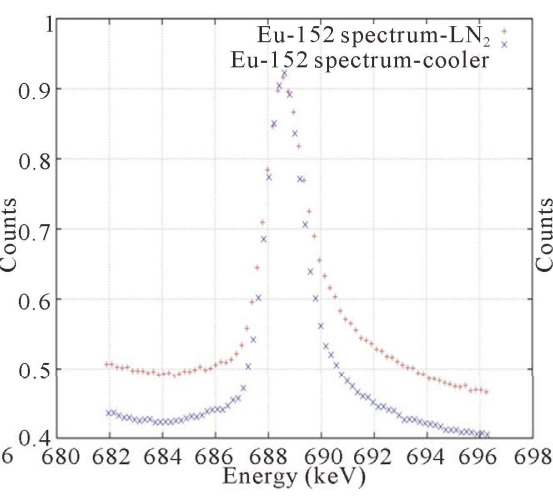

(b) Line $688.7 \mathrm{keV}$

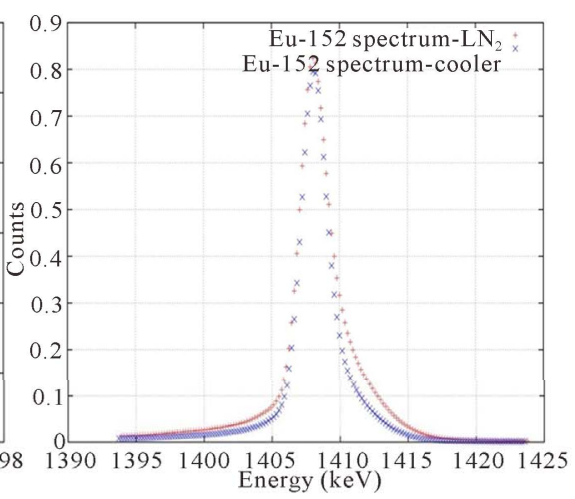

(c) Line $1408.0 \mathrm{keV}$

Figure 5. The detailed shapes of characteristic lines of ${ }^{152} \mathrm{Eu}$, from low to high energy region, namely, $244.7 \mathrm{keV}, 688.7 \mathrm{keV}$, and $1408.0 \mathrm{keV}$.

relevant studies are planned for the new running year.

\section{Acknowledgements}

This work is supported by National Natural Science Foundation of China (NSFC) under contracts No.: 11375206, 10775142, 11235011, Y21134005C, and 10825524.

\section{REFERENCES}

[1] J. Q. Wang, L. Ma, Q. Qin and C. Zhang, "Status and Performance of BEPCII", Proceedings of IPAC, Kyoto, 2010, pp. 2359-2363.

[2] Preliminary Design Report of Accelerator BEPC, Second version, 2003, in Chinese.

http://acc-center.ihep.ac.cn/bepcii/bepcii.htm

[3] M. Ablikim et al. (BESIII Collaboration), "Design and Construction of the BESIII Detector," Nuclear Instruments and Methods in Physics Research Section A: Accelerators, Spectrometers, Detectors and Associated Equipment, Vol. 614, No. 3, 2010, pp. 345-399. http://dx.doi.org/10.1016/j.nima.2009.12.050

[4] K.-T. Chao and Y.-F. Wang, "BESIII Physics," International Journal of Modern Physics A, Vol. 24, Suppl. 1, 2009, p. 1.

[5] C.-D. Fu and X.-H. Mo, "Significance of Absolute Energy Scale for Physics at BESIII," Chinese Physics C, 2008, Vol. 32, No. 10, pp. 776-780. http://dx.doi.org/10.1088/1674-1137/32/10/002

[6] X.-H. Mo, "Study of High Precision $\tau$ Mass Measurement at BESIII," Nuclear Physics B-Proceedings Supplements, Vol. 169, 2007, pp. 132-139.

http://dx.doi.org/10.1016/j.nuclphysbps.2007.02.107

[7] Y. K. Wang, X. H. Mo, C. Z. Yuan and J. P. Liu, "Optimization of the Data Taking Strategy for a High Precision $\tau \tau$ Mass Measurement," Nuclear Instruments and Methods in Physics Research Section A: Accelerators, Spectrometers, Detectors and Associated Equipment, Vol. 583, No. 2-3, 2007, pp. 479-484.

http://dx.doi.org/10.1016/j.nima.2007.09.026

[8] M. N. Achasov, "Energy Determination at BEPC-II,"
Nuclear Physics B-Proceedings Supplements, Vol. 189, 2009, pp. 366-370.

http://dx.doi.org/10.1016/j.nuclphysbps.2009.03.059

[9] Mo, X.-H., et al., "Beam Energy Measurement System at BEPC II," Chinese Physics C, Vol. 34, No. 6, 2010, pp. 912-917. http://dx.doi.org/10.1088/1674-1137/34/6/050

[10] E. V. Abakumova, "The Beam Energy Measurement System for the Beijing Electron Positron Collider," $\mathrm{Nu}$ clear Instruments and Methods in Physics Research Section A: Accelerators, Spectrometers, Detectors and Associated Equipment, Vol. 659, pp. 21-29.

[11] J. Y. Zhang, "The Beam Energy Measurement System for the Beijing Electron-Positron Collider," Nuclear Physics $B$-Proceedings Supplements, Vol. 225-227, 2012, pp. 309314. http://dx.doi.org/10.1016/j.nuclphysbps.2012.02.064

[12] X.-H. Mo, C.-D. Fu, J.-Y. Zhang and Q. Qin, et al., "Working Principles of the Energy Measurement System at BEPCII," Chinese Physics C, Vol. 32, No. 12, 2008, pp. 995-1002. http://dx.doi.org/10.1088/1674-1137/32/12/011

[13] G. F. Knoll, "Radiaiton of Detectrion and Measurement," John Wiley \& Sons, New York, 1979.

[14] User's Manual, X-Cooler II Mechanical Cooler for HPGe Detector, ORTEC.

[15] D.Gin, et al., ITR/P5-39.

[16] E. E. Haller, "Detector Materials: Germanium and Silicon," IEEE Transactions on Nuclear Science, Vol. 29, No. 3, 1982, pp. 1109-1108. http://dx.doi.org/10.1109/TNS.1982.4336330

[17] Y Y. F. Xia, X. B. Ni and Y. Q. Peng, “ Applied Methods for Experimental Nuclear Physics”, Science Press, 1989, in Chinese

[18] GLP Series Planar HPGeLow-Energy Detector Product Configuration Guide. www.ortec-online.com/download/glp.pdf.

[19] User's Manual, GEM Series-HPGe Coaxial Detector System, ORTEC

[20] http://www.wisegeek.com/what-is-a-cryocooler.htm

[21] G. N. Martin and M. R. Burke, "Modular Photon Detector Cryostat Assembly and System," US Patent No. 4851684.

[22] E. L. Grigorescu, "Standardization of ${ }^{152} \mathrm{Eu}$," Proceedings 
of the Conference on Radionuclide Metrology and Its Applications, Vol. 56, No. 1-2, 2002, pp. 435-439.

[23] Q. L. Yang, "Preparation of Standard Radioactive Solution of ${ }^{152}$ Eu," Journal of Nuclear and Radiochemistry,
Vol. 34, No.4, 2012, pp. 223-228.

[24] S. Li, "Application of Nuclear Spectroscopy," Chinese Science and Technology Press, Beijing, 1994, in Chinese. 